\title{
Assessment of a Cationic Emulsion to Control the Tear Film Evaporation Rate
}

\author{
Ali Abusharha \\ Cornea Research Chair, Department of \\ Optometry, College of Applied Medical \\ Sciences, King Saud University, Riyadh, \\ I I433, Saudi Arabia
}

Purpose: To investigate the effect of a single application of cationic emulsion in controlling tear film evaporation and improving tear quality and quantity.

Materials a Methods: Twenty male subjects diagnosed with DE were enrolled in the study with an average age of $45.8 \pm 6.37$ years. The tear film parameters were observed at several time points post-instillation of the cationic emulsion $(10,20,30$, and $60 \mathrm{~min})$. The tear evaporation rate (TER) was measured with a VapoMeter. Noninvasive tear break-up time and meniscus height were assessed using OCULUS Keratograph.

Results: TER decreased by more than $20 \%$ at 20,30 , and 60 minutes time points after instillation of single drop of cationic emulsion. Also, a significant improvement in tear film stability was found at all time points following the instillation of cationic emulsion eye drops. The mean tear break-up time increased from $5.55 \pm 2.87$ to $6.6 \pm 4.2 \mathrm{sec}$ at 60 minutes. The maximum increase in tear break-up time occurred at 30 minutes time point. The TMH was also significantly higher post-instillation of oil emulsion eye drops. There was a significant increase in the TMH post-therapy with oil drop at all time points.

Conclusion: The overall study findings of this study illustrate that the single application of a cationic emulsion effectively controls tear film evaporation in patients with mild to moderate DEs. The cationic emulsion efficiently enhanced both the tear film stability and the tear meniscus volume.

Keywords: dry eye, evaporation rate, cationic emulsion, tear stability

\section{Introduction}

Dry eye (DE) is a complex multifactorial disease associated with disruption of the tear film's homeostasis, resulting in many changes in the ocular surface. Factors including eyelid and blinking abnormality or tear film deficiencies represent the most common reason for the loss of tear film homeostasis, inducing tear film instability and tear hyperosmolarity. ${ }^{1}$ Up to $30 \%$ of the global population has reported a combination of symptoms and signs of DE disease. However, based on clinical findings, a prevalence rate of up to $68 \%$ has been reported in populations over 40 years old. ${ }^{2}$

The tear film lipid layer plays a vital role in controlling tear film evaporation and maintaining tear film stability. Korb et al observed the lipid layer after blinking and found that the distribution and increase in lipid secretion are blink-dependent. ${ }^{3}$ Furthermore, McCulley and Shine suggested that tear lipid has two phases. The outer thick non-polar layer protects from evaporation, whereas the thin inner polar lipid plays an important role in maintaining stability and providing interference with the aqueous layer. ${ }^{4}$
Correspondence: Ali Abusharha Department of Optometry, College of Applied Medical Sciences, King Saud University, P.O. Box 10219, Riyadh, I I433, Saudi Arabia

Email aabusharha@ksu.edu.sa 
Numerous therapeutic protocols have been adopted to control tear evaporation by increasing tear film stability. Artificial tears and lubricants are considered one of the most common methods to treat dry the signs and symptoms of DE. ${ }^{5}$ Formulations containing electrolytes, mucomimetic agents, or oil emulsion are significantly helpful in DE management. ${ }^{6}$ However, the main drawback of most of these formulations is their short retention time, as the solution can easily drain via the lacrimal drainage system. ${ }^{7}$

One type of formulation is the oil-in-water emulsion, designed to enhance, and thicken the tear film lipid layer. The first oil-in-water emulsion was combined with an antiinflammatory agent (cyclosporin). ${ }^{8}$ Then, this ophthalmic emulsion was modified to an over-the-counter oil-in-water -emulsion tear supplement with no anti-inflammatory agents. Different oils such as castor oil, mineral oil, soya bean oil, and phospholipid liposomal have been used to develop oil-in-water emulsions to manage DE signs and symptoms. ${ }^{5,9}$

The safety and efficacy of oil emulsion artificial tears in managing DE signs and symptoms are well documented. Previous studies have reported a significant improvement in corneal staining, tear stability, and production following the use of oil ophthalmic emulsion. ${ }^{10,11}$ More specifically, it has been reported that a single dose of oil emulsion resulted in a significant increase in tear meniscus height (TMH) up to 30 minutes after instilling the drop. ${ }^{11}$ Moreover, a clinical trial evaluating the efficacy of an oilbased formula (Castor oil) has shown a significant reduction in the respective signs and symptoms, including tear break-up time, corneal staining, and tear production following the use of the formula for seven days. ${ }^{10}$ It has been shown that optical coherence tomography imaging (OCT) has the potential to provide clinicians with a reliable approach to evaluate rapidly the preocular tear film thinning and adhesive properties of the ocular surface. ${ }^{12,13}$ A previous study evaluated the clearance of tear lipids using OCT imaging has reported a prolonged tear lipid retention time following installation of oil-in-water emulsion. $^{14}$

Excessive tear evaporation rate is considered a major cause of DE symptoms, leading to tear hyperosmolarity. Tear hyperosmolarity causes inflammatory processes in epithelial cells. These processes activate the inflammatory cells to generate cytokines and matrix metalloproteinases. ${ }^{15}$ The Definition and Classification Subcommittee of the International Workshop on Dry Eye (DEWS) suggested that all forms of DE, either aqueous deficient DE (ADDE) or evaporative dry eye are evaporative. ${ }^{1}$ Therefore, managing and controlling tear film evaporation is vital in treating the signs and symptoms of DE. Although several research studies have been performed on lipid containing artificial tears, no previous studies have investigated the immediate effect of cationic emulsion (oil-based formulation) in controlling tear film evaporation. This study investigated the effect of a single application of cationic emulsion in controlling tear film evaporation and improving tear quality and quantity.

\section{Method}

Twenty male subjects diagnosed with DE were enrolled in the study with an average age of $45.8 \pm 6.37$ years. Patients were enrolled in the study if they fulfilled the following criteria: Ocular surface disease index (OSDI) score $>13$, noninvasive tear film break-up time $<10 \mathrm{~s}$ and $<10 \mathrm{~mm}$ in 5 min for Schirmer test. Subjects with a history of ocular surgery, ocular disease or who wear contact lenses were excluded from this study. Patients taking medications that could influence the tear film were also excluded from the study. All procedures were approved by the College of Applied Medical Sciences Ethics Committee, King Saud University, Riyadh, Saudi Arabia. All subjects provided written informed consent. The study was conducted according to the tenets of the Declaration of Helsinki.

\section{Tear Parameters}

\section{Tear Evaporation Rate (TER)}

The TER was measured with a VapoMeter (Delfin Technologies, Kuopio, Finland) [19]. Initially, two readings were obtained with eyes open, representing the evaporation rate from the ocular surface and the lid skin. Then, second measurement was obtained with eyes closed, and these measurements were subtracted from the openeye readings. Three measurements were obtained; then, the average value was calculated.

\section{Noninvasive Tear Break-Up Time and Meniscus Height}

The OCULUS Keratograph 4 (OCULUS Inc., Wetzlar, Germany) was used to monitor the change in the (NITBUT) and TMH post-instillation. The Keratograph illumination system consists of 200 red LEDs (wavelength $653 \mathrm{~nm}$ ). It is installed with a Placido disk consisting of 22 rings which project an illuminated ring pattern on the corneal surface. ${ }^{16}$ Furthermore, the Keratograph 


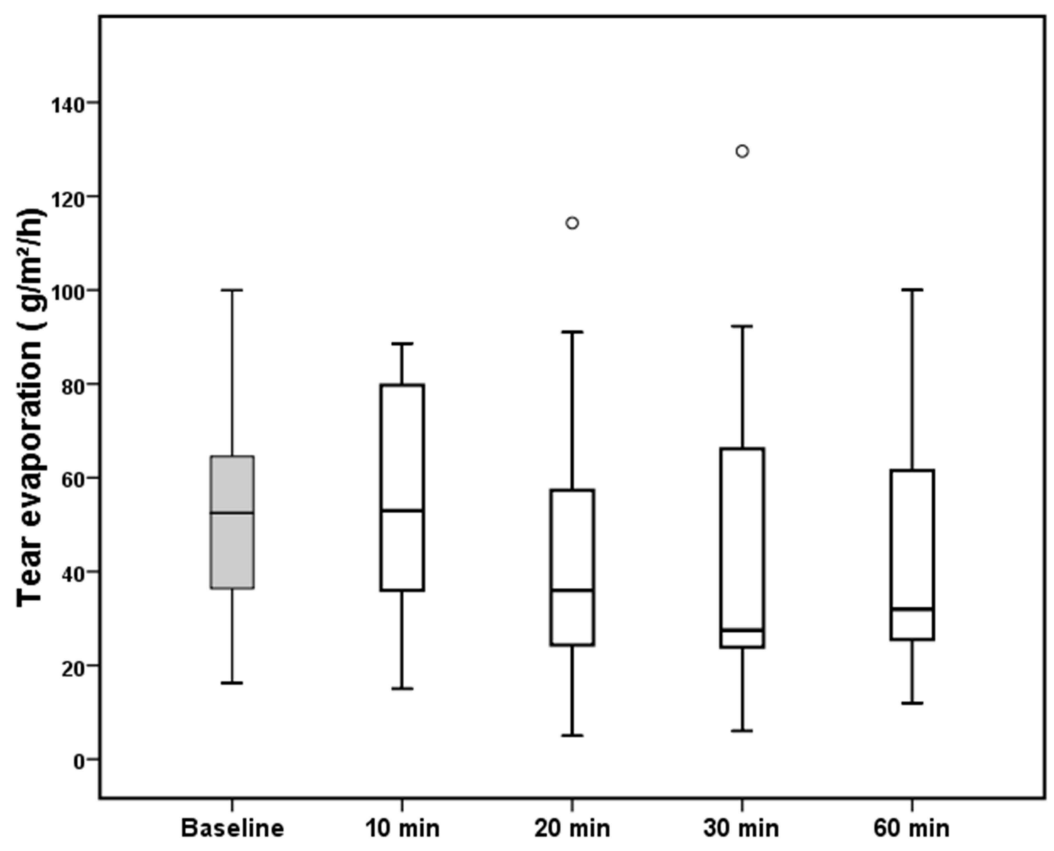

Figure I A box plot showing evaporation rate measured at different time points pre and post instillation of cationic emulsion.

illumination system is equipped with software (Tear Film Scan) that can provide automated analysis of the tear break-up time, the meniscus height and other ocular parameters. $^{16}$

In order to monitor changes to tear film behavior after instilling tear supplements, the tear film parameters were observed at several time points post-instillation of the cationic oil-based eye drop (10, 20, 30, and $60 \mathrm{~min})$.

\section{Statistical Analysis}

Variables were tested for normality using a KolmogorovSmirnov test. A repeated measure ANOVA and Tukey's posthoc test were applied for normally distributed data while the ordinal and data with non-normal distribution were analysed using Friedman test and post-hoc Wilcoxon rank-sum test.

\section{Results}

The mean tear film evaporation rate decreased by more than $20 \%$ at 20,30, and 60 minutes time points after instillation of single drop of cationic emulsion (Figure 1).
However, this reduction was not statistically significant. The mean tear film dropped from $40.88 \pm 37.14$ at baseline to $28.88 \pm 16.90 \mathrm{~g} / \mathrm{m}^{2} / \mathrm{h}$ after 60 minutes (Table 1 ).

Tear film stability improved significantly from baseline to 20,30 , and 60 minutes time points following the instillation of cationic emulsion eye drops $(p=0.03)$. The mean tear break-up time increased from $5.55 \pm 2.87$ to $6.6 \pm 4.2$ sec at 60 minutes (Figure 2). The maximum increase in tear break-up time occurred at 30 minutes time point, with an average value of $7.57 \pm 3.94 \sec (p=0.01)$ (Table 1$)$. Figure 2 demonstrates the changes in tear break-up time measured at different time points before and after instillation of cationic emulsion eye drops.

The TMH was significantly higher post-instillation of cationic emulsion eye drops $(p=0.004)$. There was a significant increase in the TMH post-therapy with cationic emulsion at all time points (10, 20, 30 and 60 minutes) compared to the values obtained at baseline $(p=0.01, \mathrm{p}=$ $0.007, p=0.018$ and $p=0.05$, respectively) (Figure 2). The maximum increase in $\mathrm{TMH}$ was recorded at time

Table I Mean and Standard Deviation of the Tear Evaporation Rate $\left(g / M^{2} / h\right)$ Tear Break-Up Time (Sec) and Meniscus Height (Mm) Measured Before and at Different Time Points After Instillation of Cationic Emulsion

\begin{tabular}{|l|l|l|l|l|l|}
\hline & \multicolumn{1}{|c|}{ Baseline } & \multicolumn{1}{c|}{ 10 Minutes } & \multicolumn{1}{|c|}{ 20 Minutes } & \multicolumn{1}{c|}{ 30 Minutes } & \multicolumn{1}{c|}{$\mathbf{6 0}$ Minutes } \\
\hline Tear evaporation & $40.89 \pm 37.14$ & $39.15 \pm 24.79$ & $32.27 \pm 19.62$ & $29.76 \pm 18.21$ & $28.81 \pm 16.81$ \\
Tear break-up time & $5.88 \pm 2.87$ & $4.94 \pm 2.06$ & $7.38 \pm 3.93$ & $7.57 \pm 3.06$ & $6.67 \pm 4.22$ \\
Tear meniscus height & $0.148 \pm .0186$ & $0.169 \pm .0216$ & $0.185 \pm .0179$ & $0.163 \pm .0169$ & $0.161 \pm .021$ \\
\hline
\end{tabular}




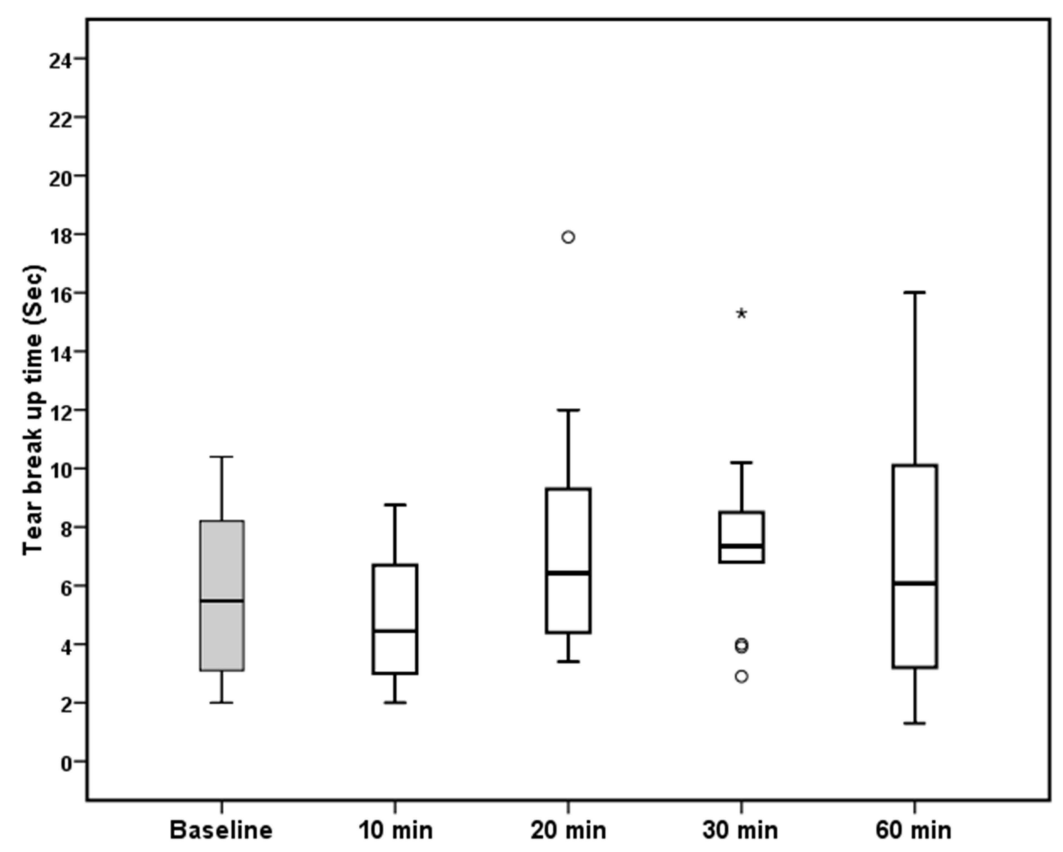

Figure $2 \mathrm{~A}$ box plot showing tear break up time measured at different time points pre and post instillation of cationic emulsion.

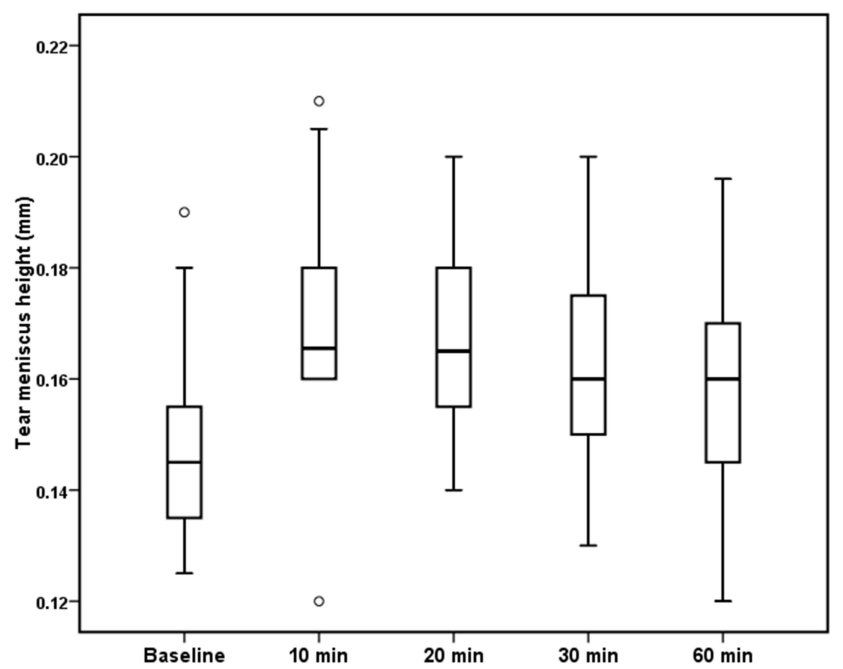

Figure 3 A box plot showing tear meniscus height measured at different time points pre and post instillation of cationic emulsion.

points 10 and 30 minutes. The mean TMH increased from 0.15 at baseline to 0.17 and $0.19 \mathrm{~mm}$ at time points 10 and 30 after, respectively, following the instillation of eye drops (Figure 3).

\section{Discussion}

The purpose of this study was to evaluate the efficiency of a single dose of cationic emulsion drop in controlling tear film evaporation and improving tear film stability. A panel of tear film measures were carried out to observe tear film parameters before and after instillation of cationic emulsion eye drops at different time points. Improvement in DE signs and symptoms has been previously reported following the application of cationic emulsion eye lubricants. ${ }^{17}$

Different tear film supplements containing various ingredients have been developed to control the signs and symptoms of DE. However, these tear film supplements are not meant to treat DE syndrome, and thus they can relieve DE signs and symptoms and help to restore the normal homeostatic state of the ocular surface. ${ }^{18}$ In addition, these formulations play a role in improving turnover, stability, and evaporation rate of the tear film and inhibiting the inflammatory changes in ocular tissue. ${ }^{19,20}$

In the present study, a cationic emulsion was used. Nanotechnologies are currently used to improve the retention and bio adhesive properties of ophthalmic solutions. A novel technology exploits the fact that the ocular surface, including the corneal and conjunctival tissues, is negatively charged. Therefore, the bio adhesive properties and retention time can be improved by using positively charged ophthalmic solutions. ${ }^{17,21}$ The efficacy of this relatively new technology in extending the ocular solution retention time is well documented. ${ }^{21}$ However, according to the TFOS DEWS II management and therapy report, the long-term safety of Cationicbased formulation needs to be assessed due to the reported alterations of the corneal stroma and corneal 
epitheliopathy. ${ }^{6}$ This affect could be caused by its preservative cetalkonium chloride or due to the fact that the prolonged bioavailability of Cationic formulation may affect the epithelial cells migration onto stromal areas of erosions that result in corneal epitheliopathy. ${ }^{22}$

This study shows that a single drop of cationic emulsion results in a noticeable reduction in tear film evaporation following the application of a cationic emulsion. More specifically, a 20 and $30 \%$ decrease in tear film evaporation was found at 20 and 60 minute time points post-instillation, respectively. This is consistent with the findings of a previous study that reported a reduction of more than $50 \%$ in tear evaporation following the use of oil emulsion for 30 days. Another study has reported that a single dose of oil-in-water emulsion can result in significant tear film evaporation. $^{23}$ This is unsurprising because the oil emulsion formulations are prepared to replenish the tear film lipid layer that plays an important role in improving tear film stability and controlling tear evaporation.

In addition, there was a significant improvement in tear film stability post-instillation of a single oil emulsion drop. An increase in tear break-up time by up to 1.7 seconds $(22 \%)$ occurred after a single dose. It has been previously demonstrated that the use of oil-based lubricants resulted in a $40 \%$ increase in the tear break-up time in patients with mild to moderate DE symptoms. ${ }^{24}$ A recent study found that the tear break-up time increased significantly when using oil-based lubricants for 35 days. ${ }^{25}$ The authors suggested that the oil lubricant formulations containing polar phospholipids can improve the interference between the lubricant components and the non-polar lipid and aqueous layer. $^{25}$

Lipid layer of tear film play plays an important role in controlling tear film evaporation and improving tear stability. Studies have suggested that tear lipids, containing proteins that interact with meibomian lipids, form a thin polar phase that maintains a stable spread of lipids over the aqueous layer. ${ }^{26}$ In addition, nanoemulsion ocular solutions could increase the lipid layer thickness at 10 minutes post-instillation of the emulsion. ${ }^{27}$ A recent study investigating tear lipid thickness using the tear film interferometry method found that the tear lipid thickness increased significantly with lipid tear supplements. ${ }^{28}$ Therefore, this increase in lipid layer thickness may explain the reduction in tear film evaporation rate and improved tear film stability.

The overall study findings of this study illustrate that the single application of a cationic emulsion effectively controls tear film evaporation in patients with mild to moderate DEs. The cationic emulsion efficiently enhanced both the tear film stability and the tear meniscus volume. These outcomes suggest that improving tear lipids spread and stability with cationic emulsions can help patients maintain a healthy and stable tear film and thus restore the normal homeostatic state of the ocular surface.

\section{Acknowledgments}

The author extends his appreciation to the College of Applied Medical Sciences Research Center and the Deanship of Scientific Research at King Saud University, for funding this research.

\section{Disclosure}

The author reports no conflicts of interest in this work.

\section{References}

1. Craig JP, Nichols KK, Akpek EK, et al. TFOS DEWS II definition and classification report. Ocul Surf. 2017;15(3):276-283. doi:10.1016/j.jtos.2017.05.008

2. Willcox MD, Argüeso P, Georgiev GA, et al. TFOS DEWS II tear film report. Ocul Surf. 2017;15(3):366-403.

3. Korb DR, Association BCL. The tear film: structure, function, and clinical examination: butterworth-heinemann. 2002.

4. McCulley JP, Shine WE. The lipid layer of tears: dependent on meibomian gland function. Exp Eye Res. 2004;78(3):361-365. doi:10.1016/S0014-4835(03)00203-3

5. Agarwal P, Craig JP, Rupenthal ID. Formulation considerations for the management of dry eye disease. Pharmaceutics. 2021;13(2):207.

6. Jones L, Downie LE, Korb D, et al. TFOS DEWS II management and therapy report. Ocul Surf. 2017;15(3):575-628.

7. Gagliano C, Papa V, Amato R, Malaguarnera G, Avitabile T. Measurement of the retention time of different ophthalmic formulations with ultrahigh-resolution optical coherence tomography. Curr Eye Res. 2018;43(4):499-502. doi:10.1080/02713683.2017.1418893

8. Stevenson D, Tauber J, Reis BL. Efficacy and safety of cyclosporin A ophthalmic emulsion in the treatment of moderate-to-severe dry eye disease: a dose-ranging, randomized trial. Ophthalmology. 2000;107(5):967-974. doi:10.1016/S0161-6420(00)00035-X

9. Garrigue J-S, Amrane M, Faure M-O, Holopainen JM, Tong L. Relevance of lipid-based products in the management of dry eye disease. J Ocul Pharmacol Ther. 2017;33(9):647-661. doi:10.1089/ jop.2017.0052

10. Khanal S, Tomlinson A, Pearce EI, Simmons PA. Effect of an oil-inwater emulsion on the tear physiology of patients with mild to moderate dry eye. Cornea. 2007;26(2):175-181. doi:10.1097/ ICO.0b013e31802b492d

11. Makri OE, Tsekouras I, Mastronikolis S, Georgakopoulos CD. Shortterm effect of non-preserved cationic oil-in-water ophthalmic emulsion on tear meniscus parameters of healthy individuals in a prospective, controlled pilot study. Med Hypothesis Discovery Innovation Ophthalmol. 2021;10(1):5-10. doi:10.51329/ mehdiophthal1415

12. Napoli PE, Coronella F, Satta GM, Galantuomo MS, Fossarello M. Evaluation of the adhesive properties of the cornea by means of optical coherence tomography in patients with meibomian gland dysfunction and lacrimal tear deficiency. PLoS One. 2014;9(12): e115762. doi:10.1371/journal.pone. 0115762 
13. Napoli PE, Nioi M, d'Aloja E, Fossarello M. The bull's eye pattern of the tear film in humans during visual fixation on en-face optical coherence tomography. Sci Rep. 2019;9(1):1-9. doi:10.1038/ s41598-018-38260-5

14. Napoli PE, Coronella F, Satta GM, Fossarello M. A novel technique of contrast-enhanced optical coherence tomography imaging in evaluation of clearance of lipids in human tears. PLoS One. 2014;9(11): e109843. doi:10.1371/journal.pone.0109843

15. Lemp MA, Baudouin C, Baum J, et al. The definition and classification of dry eye disease: report of the Definition and Classification Subcommittee of the international Dry Eye workshop. Ocul Surf. 2007;5(2):75-92.

16. Best N, Drury L, Wolffsohn JS. Clinical evaluation of the Oculus Keratograph. Cont Lens Anterior Eye. 2012;35(4):171-174. doi:10.1016/j.clae.2012.04.002

17. Robert P-Y, Cochener B, Amrane M, et al. Efficacy and safety of a cationic emulsion in the treatment of moderate to severe dry eye disease: a randomized controlled study. Eur J Ophthalmol. 2016;26 (6):546-555. doi:10.5301/ejo.5000830

18. Lemp MA. Advances in understanding and managing dry eye disease. Am J Ophthalmol. 2008;146(3):350-356. doi:10.1016/j. ajo.2008.05.016

19. Mathers WD. Why the eye becomes dry: a cornea and lacrimal gland feedback model. CLAO j. 2000;26(3):159-165.

20. McCann LC, Tomlinson A, Pearce EI, Papa V. Effectiveness of artificial tears in the management of evaporative dry eye. Cornea. 2012;31(1):1. doi:10.1097/ICO.0b013e31821b71e6

21. Daull P, Amrane M, Ismail D, et al. Cationic emulsion-based artificial tears as a mimic of functional healthy tear film for restoration of ocular surface homeostasis in dry eye disease. J Ocul Pharmacol Ther. 2020;36(6):355-365. doi:10.1089/jop.2020.0011
22. Pinheiro R, Panfil C, Schrage N, Dutescu RM. Comparison of the lubricant eyedrops Optive ${ }^{\circledR}$, Vismed Multi ${ }^{\circledR}$, and Cationorm ${ }^{\circledR}$ on the corneal healing process in an ex vivo model. Eur J Ophthalmol. 2015;25(5):379-384. doi:10.5301/ejo.5000593

23. Pearce EI, Tomlinson A, Blades KJ, Falkenberg HK, Lindsay B, Wilson CG. Effect of an oil and water emulsion on tear evaporation rate. Adv Exp Med Biol. 2002;506(A):419-424.

24. Baudouin C, Galarreta DJ, Mrukwa-Kominek E, et al. Clinical evaluation of an oil-based lubricant eyedrop in dry eye patients with lipid deficiency. Eur J Ophthalmol. 2017;27(2):122-128. doi:10.5301/ ejo. 5000883

25. Jerkins G, Greiner JV, Tong L, et al. A comparison of efficacy and safety of two lipid-based lubricant eye drops for the management of evaporative dry eye disease. Clin Ophthalmol. 2020;14:1665. doi:10.2147/OPTH.S256351

26. Shine WE, McCulley JR. Polar lipids in human meibomian gland secretions. Curr Eye Res. 2003;26(2):89-94. doi:10.1076/ ceyr.26.2.89.14515

27. Weisenberger K, Fogt N, Fogt JS. Comparison of nanoemulsion and non-emollient artificial tears on tear lipid layer thickness and symptoms. J Optom. 2021;14(1):20-27. doi:10.1016/j. optom.2020.03.002

28. Muntz A, Marasini S, Wang MT, Craig JP. Prophylactic action of lipid and non-lipid tear supplements in adverse environmental conditions: a randomised crossover trial. Ocul Surf. 2020;18(4):920-925. doi:10.1016/j.jtos.2020.08.004
Clinical Optometry

\section{Publish your work in this journal}

Clinical Optometry is an international, peer-reviewed, open access journal publishing original research, basic science, clinical and epidemiological studies, reviews and evaluations on clinical optometry. All aspects of patient care are addressed within the journal as well as the practice of optometry including economic and business analyses. Basic and clinical research papers are published that cover

Submit your manuscript here: https://www.dovepress.com/clinical-optometry-journa

\section{Dovepress}

all aspects of optics, refraction and its application to the theory and practice of optometry. The manuscript management system is completely online and includes a very quick and fair peer-review system, which is all easy to use. Visit http://www.dovepress.com/ testimonials.php to read real quotes from published authors. 